Commun. Fac. Sci. Univ. Ank. Ser. A1 Math. Stat.

Volume 69, Number 1, Pages 394-412(2020)

DOI: $10.31801 /$ cfsuasmas. 596546

ISSN 1303-5991 E-ISSN 2618-6470

http://communications.science.ankara.edu.tr/index.php?series=A1

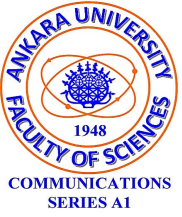

\title{
SIMPLE CRITERIA FOR UNIVALENCE AND COEFFICIENT BOUNDS FOR A CERTAIN SUBCLASSES OF ANALYTIC FUNCTIONS
}

\author{
MOSTAFA JAFARI, TEODOR BULBOACĂ, AHMAD ZIREH, \\ AND EBRAHIM ANALOUEI ADEGANI
}

\begin{abstract}
In the first part of this work we present several new geometric properties of analytic functions by applying the differential subordination. In addition, several results in the geometric functions theory pointed out. In the second part we find upper bounds for coefficients of functions in class $\mathcal{B}_{\Sigma}^{q, \mu}(\beta, \lambda, h)$ which is defined by fractional $q$-calculus operators.
\end{abstract}

\section{Introduction And Preliminaries}

Let $\mathcal{A}$ be the class of functions of the form

$$
f(z)=z+\sum_{n=2}^{\infty} a_{n} z^{n}
$$

which are analytic in the open unit disk $\mathbb{U}:=\{z \in \mathbb{C}:|z|<1\}$, and denote by $\mathcal{S}$ the class of all functions of $\mathcal{A}$ which are univalent in $\mathbb{U}$.

For two functions $f$ and $F$ which are analytic in $\mathbb{U}$, we say that the function $f$ is subordinate to $F$ in $\mathbb{U}$, and write $f(z) \prec F(z)$, if there exists a Schwarz function $\omega$, which is analytic in $\mathbb{U}$ with $\omega(0)=0$ and $|\omega(z)|<1$, such that $f(z)=F(\omega(z))$ for all $z \in \mathbb{U}$.

By Schwarz's lemma we have $|\omega(z)| \leq|z|, z \in \mathbb{U}$, which concludes that $\omega(\mathbb{U}) \subset \mathbb{U}$. Since $\omega(0)=0$ and $\omega(\mathbb{U}) \subset \mathbb{U}$ it follows that if $f(z) \prec F(z)$, then $f(0)=F(0)$ and $f(\mathbb{U}) \subset F(\mathbb{U})$. In particular, if the function $F$ is univalent in $\mathbb{U}$, then we have the following equivalence

$$
f(z) \prec F(z) \Leftrightarrow f(0)=F(0) \quad \text { and } \quad f(\mathbb{U}) \subset F(\mathbb{U}) .
$$

Received by the editors: July 25, 2019; Accepted: November 01, 2019.

2010 Mathematics Subject Classification. Primary 30C45; Secondary 30C80.

Key words and phrases. Univalent function, differential subordination, starlike functions, closeto-convex functions, bi-univalent functions, fractional $q$-calculus operators, Faber polynomials.

(C)2020 Ankara University

Communications Faculty of Sciences University of Ankara-Series A1 Mathematics and Statistics 
First, Miller and Mocanu [18 in 1978 introduced the method of differential subordinations and then in recent years several authors obtained several applications in the geometric functions theory by using differential subordination, see for example [5, 7, 8, 9, 12, 13, 15, 20.

We denote by $\mathcal{S}^{*}(\alpha)$ the subclass of $\mathcal{A}$ consisting of functions which are starlike of order $\alpha$ in $\mathbb{U}$, as follows:

$$
\mathcal{S}^{*}(\alpha):=\left\{f \in \mathcal{A}: \operatorname{Re} \frac{z f^{\prime}(z)}{f(z)}>\alpha, z \in \mathbb{U}, 0 \leq \alpha<1\right\},
$$

and, in particular, $\mathcal{S}^{*}:=\mathcal{S}^{*}(0)$ is the class of starlike functions in the unit disk $\mathbb{U}$.

Also, we denote by $\mathcal{C}(\alpha)$ the subclass of $\mathcal{A}$ consisting of functions which are close-to-convex of order $\alpha$ if there exists a function $g \in \mathcal{S}^{*}$ such that

$$
\operatorname{Re} \frac{z f^{\prime}(z)}{g(z)}>\alpha, z \in \mathbb{U}, 0 \leq \alpha<1 .
$$

In particular, $\mathcal{C}:=\mathcal{C}(0)$ is the class of close-to-convex functions in the unit disk $\mathbb{U}$. It is well-known that $\mathcal{S}(\alpha) \subset \mathcal{S}$ and $\mathcal{C}(\alpha) \subset \mathcal{S}$, for all $0 \leq \alpha<1$.

It is well known that every function $f \in \mathcal{S}$ contains a disk of radius $\frac{1}{4}$. Therefore, every function $f \in \mathcal{S}$ has an inverse $f^{-1}$, which is defined by $f^{-1}(f(z))=z(z \in \mathbb{U})$, and $f\left(f^{-1}(w)\right)=w\left(|w|<r_{0}(f) ; r_{0}(f) \geq \frac{1}{4}\right)$, where

$g(w)=f^{-1}(w)=w-a_{2} w^{2}+\left(2 a_{2}^{2}-a_{3}\right) w^{3}-\left(5 a_{2}^{3}-5 a_{2} a_{3}+a_{4}\right) w^{4}+\cdots=: w+\sum_{n=2}^{\infty} b_{n} w^{n}$.

A function $f \in \mathcal{A}$ is said to be bi-univalent in $\mathbb{U}$ if both $f$ and $f^{-1}$ are univalent in $\mathbb{U}$, and let $\Sigma$ denote the class of bi-univalent functions in $\mathbb{U}$. In recent years many authors made an effort to introduce various subclasses of the bi-univalent function class $\Sigma$, see for example [10, 22, 23, 27.

Purohit and Raina [25] (see also [22]) defined a fractional q-differential operator $\Omega_{q}^{\mu}$ (by using the definitions of the fractional $q$-calculus operators) for a function $f$ of the form 1.1 by

$$
\Omega_{q}^{\mu} f(z)=z+\sum_{n=2}^{\infty} \Psi_{q}^{n}(\mu) a_{n} z^{n}=\frac{\Gamma_{q}(2-\mu)}{\Gamma_{q}(2)} z^{\mu-1} D_{q, z}^{\mu} f(z), z \in \mathbb{U},
$$

where

$$
\Theta_{n}:=\Psi_{n}^{q}(\mu)=\frac{\Gamma_{q}(2-\mu) \Gamma_{q}(n+1)}{\Gamma_{q}(2) \Gamma_{q}(n+1-\mu)},-\infty<\mu<2,0<q<1,
$$

where $D_{q, z}^{\mu} f$ in 1.3 represents, respectively, a fractional $q$-integral of $f$ of order $\mu$ when $-\infty<\mu<0$, and a fractional $q$-derivative of $f$ of order $\mu$ when $0 \leq \mu<2$. 
We note that $\Omega_{q}^{0} f(z)=f(z)$ and $\lim _{q \rightarrow 1^{-}} \Omega_{q}^{\mu} f(z)=\Omega^{\mu} f(z)$ (see Owa and Srivastava [24, Aouf and Dziok [6] and Srivastava and Aouf [26]).

Definition 1.1. [22] Let $h: \mathbb{U} \rightarrow \mathbb{C}$ be a convex (univalent) function such that

$$
h(0)=1 \quad \text { and } \quad \operatorname{Re} h(z)>0, z \in \mathbb{U} .
$$

A function $f \in \Sigma$ given by 1.1 is said to be in the class $\mathcal{B}_{\Sigma}^{q, \mu}(\beta, \lambda, h)$ if the following conditions are satisfied:

$$
e^{i \beta}\left(\frac{z^{1-\lambda}\left(\Omega_{q}^{\mu} f(z)\right)^{\prime}}{\left[\Omega_{q}^{\mu} f(z)\right]^{1-\lambda}}\right) \prec h(z) \cos \beta+i \sin \beta
$$

and

$$
e^{i \beta}\left(\frac{w^{1-\lambda}\left(\Omega_{q}^{\mu} g(w)\right)^{\prime}}{\left[\Omega_{q}^{\mu} g(w)\right]^{1-\lambda}}\right) \prec h(w) \cos \beta+i \sin \beta,
$$

where $\beta \in\left(\frac{-\pi}{2}, \frac{\pi}{2}\right), \lambda \geq 0, z, w \in \mathbb{U}$, and where $g=f^{-1}$ is given by 1.2 .

The following lemmas will be used in prove the main result.

Lemma 1.1. [19] Let $p(z)=1+\sum_{n \geq m}^{\infty} c_{n} z^{n}, c_{m} \neq 0$, be an analytic function in $|z|<1$ with $p(0)=1$. If there exists a point $z_{0}$, with $\left|z_{0}\right|<1$, such that

$$
\operatorname{Re} p(z)>0 \text { for }|z|<\left|z_{0}\right|
$$

and

then we have

$$
\operatorname{Re} p\left(z_{0}\right)=0
$$

$$
z_{0} p^{\prime}\left(z_{0}\right)=\left\{\begin{array}{lll}
i k p\left(z_{0}\right), & \text { when } & p\left(z_{0}\right) \neq 0 \\
-l / 2, & \text { when } & p\left(z_{0}\right)=0
\end{array}\right.
$$

for some $k \geq m, l \geq m$.

Lemma 1.2. [11, p. 190] Let $u$ be analytic function in the unit disk $\mathbb{U}$, with $u(0)=0$, and $|u(z)|<1$ for all $z \in \mathbb{D}$, with the power series expansion

$$
u(z)=\sum_{n=1}^{\infty} c_{n} z^{n}, \quad z \in \mathbb{D} .
$$

Then, $\left|c_{n}\right| \leq 1$ for all $n=1,2,3, \ldots$ Furthermore, $\left|c_{n}\right|=1$ for some $n$ ( $n=$ $1,2,3, \ldots)$ if and only if $u(z)=e^{i \theta} z^{n}, \theta \in \mathbb{R}$.

Lemma 1.3. 14] Let the function $w$ be a Schwarz function with the power series expansion given by $w(z)=\sum_{n=1}^{\infty} w_{n} z^{n}, z \in \mathbb{U}$. Then, for every complex number $s$, the next inequality holds:

$$
\left|w_{2}-s w_{1}^{2}\right| \leq 1+(|s|-1)\left|w_{1}^{2}\right| .
$$


In Section 2, the paper aims in presenting several new geometric properties of analytic functions by applying the differential subordinations, and in addition, several special results are pointed out. In Section 3 we use the Faber polynomial expansion techniques to derive bounds for the coefficients $\left|a_{n}\right|$ for the functions of the class $\mathcal{B}_{\Sigma}^{q, \mu}(\beta, \lambda, h)$, that our results generalize and improve some of the previously ones. In the literature, several authors used the Faber polynomial expansions under certain conditions to determine the general coefficient bounds of $\left|a_{n}\right|$ for the analytic bi-univalent functions (see, for example, [16, 17, 30]).

\section{Sufficient Conditions for Univalence and Starlikeness}

In the following section we study differential subordinations and several sufficient conditions for the univalence, starlikeness and close-to-convexity of functions $f \in \mathcal{A}$.

Theorem 2.1. Let $p$ be an analytic function in $\mathbb{U}$, with $p(0)=1$ and $p^{\prime}(0) \neq 0$, that satisfies

$$
\operatorname{Re}\left[\frac{p(z)+\frac{z p^{\prime}(z)}{p(z)}}{a+p^{2}(z)+z p^{\prime}(z)}\right]^{2}>0, z \in \mathbb{U}, \text { for some } a \in \mathbb{R} \backslash\{-1\} .
$$

Then,

$$
\operatorname{Re} p(z)>0, z \in \mathbb{U} \text {. }
$$

Proof. If $a=0$, using the fact that $p(0)=1$ it is easy to prove that the assumption (2.1) implies $\operatorname{Re} p(z)>0, z \in \mathbb{U}$, and therefore we will assume that $a \neq 0$. Also, since the inequality 2.1 holds for $z_{*}=0$, it is necessary to assume that $a \neq-1$.

Supposing that there exists a point $z_{0} \in \mathbb{U}$ such that

$$
\operatorname{Re} p(z)>0, \text { for }|z|<\left|z_{0}\right|
$$

and

$$
\operatorname{Re} p\left(z_{0}\right)=0
$$

it follows that

$$
p\left(z_{0}\right)=i \lambda, \lambda \in \mathbb{R} .
$$

Hence, according to Lemma 1.1 for $m=1$, we have

$$
z_{0} p^{\prime}\left(z_{0}\right)=\left\{\begin{array}{lll}
i k p\left(z_{0}\right), & \text { when } & p\left(z_{0}\right) \neq 0 \\
-l / 2, & \text { when } & p\left(z_{0}\right)=0
\end{array}= \begin{cases}-k \lambda, & \text { when } \lambda \neq 0 \\
-l / 2, & \text { when } \lambda=0\end{cases}\right.
$$

for some $k \geq 1, l \geq 1$.

(i) For the case $p\left(z_{0}\right) \neq 0$ suppose that

$$
a+p^{2}\left(z_{0}\right)+z p^{\prime}\left(z_{0}\right)=a-\lambda^{2}-k \lambda=0 .
$$

$(\alpha)$ If 


$$
p\left(z_{0}\right)+\frac{z_{0} p^{\prime}\left(z_{0}\right)}{p\left(z_{0}\right)}=i(\lambda+k) \neq 0,
$$

then $z_{0} \in \mathbb{U}$ will be a double pole for the function

$$
\left[\frac{p(z)+\frac{z p^{\prime}(z)}{p(z)}}{a+p^{2}(z)+z p^{\prime}(z)}\right]^{2}
$$

and therefore, in any neighborhood $U\left(z_{0} ; \rho\right):=\{z \in \mathbb{C}:|z|<\rho\} \subset \mathbb{U}$ of the pole $z_{0}$ there exists at least a $z_{\rho} \in U\left(z_{0} ; \rho\right)$ such that

$$
\operatorname{Re}\left[\frac{p\left(z_{\rho}\right)+\frac{z_{\rho} p^{\prime}\left(z_{\rho}\right)}{p\left(z_{\rho}\right)}}{a+p^{2}\left(z_{\rho}\right)+z_{\rho} p^{\prime}\left(z_{\rho}\right)}\right]^{2}<0
$$

which contradicts the assumption (2.1).

$(\beta)$ If

$$
p\left(z_{0}\right)+\frac{z_{0} p^{\prime}\left(z_{0}\right)}{p\left(z_{0}\right)}=i(\lambda+k)=0,
$$

from this relation and $(2.2)$ it follows that $a=0$, which contradicts our assumption.

Therefore, from $(\alpha)$ and $(\beta)$ we deduce that the assumption (2.1) implies that the function

$$
\left[\frac{p(z)+\frac{z p^{\prime}(z)}{p(z)}}{a+p^{2}(z)+z p^{\prime}(z)}\right]^{2}
$$

is analytic in $\mathbb{U}$, and

$$
\operatorname{Re}\left[\frac{p\left(z_{0}\right)+\frac{z_{0} p^{\prime}\left(z_{0}\right)}{p\left(z_{0}\right)}}{a+p^{2}\left(z_{0}\right)+z p^{\prime}\left(z_{0}\right)}\right]^{2}=\operatorname{Re}\left(\frac{i \lambda+i k}{a-\lambda^{2}-k \lambda}\right)^{2}=-\left(\frac{\lambda+k}{a-\lambda^{2}-k \lambda}\right)^{2} \leq 0
$$

which is a contradiction with the assumption (2.1).

(ii) For the case $p\left(z_{0}\right)=0$ it follows that $z_{0} p^{\prime}\left(z_{0}\right)$ is a negative real number, and the function $\frac{z p^{\prime}(z)}{p(z)}$ has a simple pole at $z_{0}$. Since $p(0)=1$, then $z_{0} \in \mathbb{U} \backslash\{0\}$ will 
be at least a double pole for the function

$$
\left[\frac{p(z)+\frac{z p^{\prime}(z)}{p(z)}}{a+p^{2}(z)+z p^{\prime}(z)}\right]^{2}
$$

and therefore, in any neighborhood $U\left(z_{0} ; \rho\right):=\{z \in \mathbb{C}:|z|<\rho\} \subset \mathbb{U}$ of the pole $z_{0}$ there exists at least a $z_{\rho} \in U\left(z_{0} ; \rho\right)$ such that have

$$
\operatorname{Re}\left[\frac{p\left(z_{\rho}\right)+\frac{z_{\rho} p^{\prime}\left(z_{\rho}\right)}{p\left(z_{\rho}\right)}}{a+p^{2}\left(z_{\rho}\right)+z_{\rho} p^{\prime}\left(z_{\rho}\right)}\right]^{2}<0
$$

which contradicts the assumption (2.1).

Concluding, from the above cases it follows that $\operatorname{Re} p(z)>0$ for all $z \in \mathbb{U}$, and the proof of the theorem is complete.

For $f \in \mathcal{A}$ and $p:=f^{\prime}$ the above theorem leads to the following result which gives sufficient condition for the close-to-convexity (univalence) of the function $f$ :

Corollary 2.1. If $f \in \mathcal{A}$, with $f^{\prime \prime}(0) \neq 0$, and satisfies

$$
\operatorname{Re}\left[\frac{f^{\prime}(z)+\frac{z f^{\prime \prime}(z)}{f^{\prime}(z)}}{a+\left[f^{\prime 2}+z f^{\prime \prime}(z)\right.}\right]^{2}>0, z \in \mathbb{U}, \quad \text { for some } a \in \mathbb{R} \backslash\{-1\}
$$

then

$$
\operatorname{Re} f^{\prime}(z)>0, z \in \mathbb{U} \text {. }
$$

For $f \in \mathcal{A}$ and $p(z):=\frac{z f^{\prime}(z)}{f(z)}$, then $p^{\prime}(0) \neq 0$ is equivalent to $f^{\prime \prime}(0) \neq 0$, and Theorem 2.1 leads to the following result which gives a sufficient starlikeness (univalence) condition:

Corollary 2.2. If $f \in \mathcal{A}$, with $f^{\prime \prime}(0) \neq 0$, and satisfies

$$
\operatorname{Re}\left[\frac{1+\frac{z f^{\prime \prime}(z)}{f^{\prime}(z)}}{a+\frac{z f^{\prime}(z)}{f(z)}+\frac{z^{2} f^{\prime \prime}(z)}{f(z)}}\right]^{2}>0, z \in \mathbb{U}, \text { for some } a \in \mathbb{R} \backslash\{-1\},
$$

then

$$
\operatorname{Re} \frac{z f^{\prime}(z)}{f(z)}>0, z \in \mathbb{U}
$$


Theorem 2.2. Let $p$ be an analytic function in $\mathbb{U}$, with $p(0)=1, p^{\prime}(0) \neq 0$, that satisfies

$$
\operatorname{Re}\left[\frac{p(z)+\frac{z p^{\prime}(z)}{p(z)}}{a+\frac{z p^{\prime}(z)}{p^{2}(z)}}\right]^{2}>0, z \in \mathbb{U}, \text { for some } a \in \mathbb{R} \backslash\{0\}
$$

Then,

$$
\operatorname{Re} p(z)>0, z \in \mathbb{U} .
$$

Proof. Since the inequality $(2.3)$ holds for $z_{*}=0$ it is necessary to assume that $a \neq 0$. For $a=1$, using the fact that $p(0)=1$ it is easy to prove that the assumption (2.3) implies our conclusion, and thus we will assume that $a \neq 1$.

Supposing that there exists a point $z_{0} \in \mathbb{U}$ such that

$$
\operatorname{Re} p(z)>0, \text { for }|z|<\left|z_{0}\right|
$$

and

$$
\operatorname{Re} p\left(z_{0}\right)=0
$$

it follows that

$$
p\left(z_{0}\right)=i \lambda, \lambda \in \mathbb{R} .
$$

Now, using Lemma 1.1 for $m=1$, we have

$$
z_{0} p^{\prime}\left(z_{0}\right)=\left\{\begin{array}{lll}
i k p\left(z_{0}\right), & \text { when } & p\left(z_{0}\right) \neq 0 \\
-l / 2, & \text { when } & p\left(z_{0}\right)=0
\end{array}= \begin{cases}-k \lambda, & \text { when } \lambda \neq 0 \\
-l / 2, & \text { when } \quad \lambda=0,\end{cases}\right.
$$

for some $k \geq 1, l \geq 1$.

(i) For the case $p\left(z_{0}\right) \neq 0$, that is $\lambda \neq 0$, suppose that

$$
a+\frac{z_{0} p^{\prime}\left(z_{0}\right)}{p^{2}\left(z_{0}\right)}=a+\frac{k}{\lambda}=0 .
$$

$(\alpha)$ If

$$
p\left(z_{0}\right)+\frac{z_{0} p^{\prime}\left(z_{0}\right)}{p\left(z_{0}\right)}=i(\lambda+k) \neq 0,
$$

then $z_{0} \in \mathbb{U}$ will be a double pole for the function

$$
\left[\frac{p(z)+\frac{z p^{\prime}(z)}{p(z)}}{a+\frac{z p^{\prime}(z)}{p^{2}(z)}}\right]^{2}
$$


and therefore, in any neighborhood $U\left(z_{0} ; \rho\right):=\{z \in \mathbb{C}:|z|<\rho\} \subset \mathbb{U}$ of the pole $z_{0}$ there exists at least a $z_{\rho} \in U\left(z_{0} ; \rho\right)$ such that

$$
\operatorname{Re}\left[\frac{p\left(z_{\rho}\right)+\frac{z_{\rho} p^{\prime}\left(z_{\rho}\right)}{p\left(z_{\rho}\right)}}{a+\frac{z_{\rho} p^{\prime}\left(z_{\rho}\right)}{p^{2}\left(z_{\rho}\right)}}\right]^{2}<0,
$$

which contradicts the assumption 2.3 .

$(\beta)$ If

$$
p\left(z_{0}\right)+\frac{z_{0} p^{\prime}\left(z_{0}\right)}{p\left(z_{0}\right)}=i(\lambda+k)=0,
$$

from this relation and (2.4) it follows that $a=1$, which contradicts our assumption.

Therefore, from $(\alpha)$ and $(\beta)$ we deduce that the assumption $(2.3)$ implies that the function

is analytic in $\mathbb{U}$, and

$$
\left[\frac{p(z)+\frac{z p^{\prime}(z)}{p(z)}}{a+\frac{z p^{\prime}(z)}{p^{2}(z)}}\right]^{2}
$$

$$
\operatorname{Re}\left[\frac{p\left(z_{0}\right)+\frac{z_{0} p^{\prime}\left(z_{0}\right)}{p\left(z_{0}\right)}}{a+\frac{z_{0} p^{\prime}\left(z_{0}\right)}{p^{2}\left(z_{0}\right)}}\right]^{2}=\operatorname{Re}\left(\frac{i \lambda+i k}{a+\frac{k}{\lambda}}\right)^{2}=-\left(\frac{\lambda+k}{a+\frac{k}{\lambda}}\right)^{2} \leq 0,
$$

which is a contradiction with the assumption $(2.3)$.

(ii) For the case $p\left(z_{0}\right)=0$, since

$$
\left[\frac{p(z)+\frac{z p^{\prime}(z)}{p(z)}}{a+\frac{z p^{\prime}(z)}{p^{2}(z)}}\right]^{2}=\left[\frac{p^{3}(z)+z p(z) p^{\prime}(z)}{a p^{2}(z)+z p^{\prime}(z)}\right]^{2},
$$

it follows that

$$
\operatorname{Re}\left[\frac{p^{3}\left(z_{0}\right)+z_{0} p\left(z_{0}\right) p^{\prime}\left(z_{0}\right)}{a p^{2}\left(z_{0}\right)+z_{0} p^{\prime}\left(z_{0}\right)}\right]^{2}=0
$$

which contradicts the assumption (2.3).

Thus, from the above cases it follows that $\operatorname{Re} p(z)>0$ for all $z \in \mathbb{U}$.

For $f \in \mathcal{A}$ and $p:=f^{\prime}$, and for $p(z):=\frac{z f^{\prime}(z)}{f(z)}$, Theorem 2.2 reduces to the following two results which represent sufficient condition for the close-to-convexity and starlikeness, respectively: 
Corollary 2.3. If $f \in \mathcal{A}$, with $f^{\prime \prime}(0) \neq 0$, and satisfies

$$
\operatorname{Re}\left[\frac{f^{\prime}(z)+\frac{z f^{\prime \prime}(z)}{f^{\prime}(z)}}{a+\frac{z f^{\prime \prime}(z)}{\left[f^{\prime 2}\right.}}\right]^{2}>0, z \in \mathbb{U}, \text { for some } a \in \mathbb{R} \backslash\{0\},
$$

then,

$$
\operatorname{Re} f^{\prime}(z)>0, z \in \mathbb{U} \text {. }
$$

Corollary 2.4. If $f \in \mathcal{A}$, with $f^{\prime \prime}(0) \neq 0$, and satisfies

$$
\operatorname{Re}\left[\frac{1+\frac{z f^{\prime \prime}(z)}{f^{\prime}(z)}}{a+\frac{f(z)}{z f^{\prime}(z)}\left(1+\frac{z f^{\prime \prime}(z)}{f^{\prime}(z)}-\frac{z f^{\prime}(z)}{f(z)}\right)}\right]^{2}>0, z \in \mathbb{U}, \text { for some } a \in \mathbb{R} \backslash\{0\},
$$

then,

$$
\operatorname{Re} \frac{z f^{\prime}(z)}{f(z)}>0, z \in \mathbb{U}
$$

Theorem 2.3. Let $p$ be an analytic function in $\mathbb{U}$, with $p(0)=1, p^{\prime}(0) \neq 0$, that satisfies

$$
\operatorname{Re}\left[\frac{p(z)\left[a+z p^{\prime}(z)\right]}{a+p^{2}(z)+z p^{\prime}(z)}\right]^{2}>0, z \in \mathbb{U}, \quad \text { for some } \quad a \in\left(-\infty, \frac{1}{2}\right) \backslash\{-1,0\} .
$$

Then

$$
\operatorname{Re} p(z)>0, z \in \mathbb{U} .
$$

Proof. First, since the assumption 2.5 holds for $z_{*}=0$, it is necessary to assume that $a \neq 0$ and $a \neq-1$. If we suppose that there exists a point $z_{0} \in \mathbb{U}$ such that

$$
\operatorname{Re} p(z)>0, \text { for }|z|<\left|z_{0}\right|
$$

and

it follows that

$$
\operatorname{Re} p\left(z_{0}\right)=0
$$

$$
p\left(z_{0}\right)=i \lambda, \lambda \in \mathbb{R} .
$$

Hence, according to Lemma 1.1 for $m=1$, we have

$$
z_{0} p^{\prime}\left(z_{0}\right)=\left\{\begin{array}{lll}
i k p\left(z_{0}\right), & \text { when } & p\left(z_{0}\right) \neq 0 \\
-l / 2, & \text { when } & p\left(z_{0}\right)=0
\end{array}= \begin{cases}-k \lambda, & \text { when } \lambda \neq 0 \\
-l / 2, & \text { when } \quad \lambda=0,\end{cases}\right.
$$

for some $k \geq 1, l \geq 1$.

(i) For the case $p\left(z_{0}\right) \neq 0$, that is $\lambda \neq 0$, suppose that

$$
a+p^{2}\left(z_{0}\right)+z p^{\prime}\left(z_{0}\right)=a-\lambda^{2}-k \lambda=0 .
$$

$(\alpha)$ If

$$
p\left(z_{0}\right)\left[a+z_{0} p^{\prime}\left(z_{0}\right)\right]=i \lambda(a-k \lambda) \neq 0,
$$


then $z_{0} \in \mathbb{U}$ will be a double pole for the function

$$
\left[\frac{p(z)\left[a+z p^{\prime}(z)\right]}{a+p^{2}(z)+z p^{\prime}(z)}\right]^{2}
$$

and therefore, in any neighborhood $U\left(z_{0} ; \rho\right):=\{z \in \mathbb{C}:|z|<\rho\} \subset \mathbb{U}$ of the pole $z_{0}$ there exists at least a $z_{\rho} \in U\left(z_{0} ; \rho\right)$ such that have

$$
\operatorname{Re}\left[\frac{p\left(z_{\rho}\right)\left[a+z_{\rho} p^{\prime}\left(z_{\rho}\right)\right]}{a+p^{2}\left(z_{\rho}\right)+z_{\rho} p^{\prime}\left(z_{\rho}\right)}\right]^{2}<0
$$

which contradicts the assumption $(2.5)$.

$(\beta)$ If

$$
p\left(z_{0}\right)\left[a+z_{0} p^{\prime}\left(z_{0}\right)\right]=i \lambda(a-k \lambda)=0,
$$

hence $a=k \lambda$, and from 2.6 it follows that $-\lambda^{2}=0$, that contradicts the fact $\lambda \neq 0$.

Therefore, from $(\alpha)$ and $(\beta)$ we deduce that the assumption $(2.1)$ implies that the function

is analytic in $\mathbb{U}$, and

$$
\left[\frac{p(z)\left[a+z p^{\prime}(z)\right]}{a+p^{2}(z)+z p^{\prime}(z)}\right]^{2}
$$

$$
\operatorname{Re}\left[\frac{p\left(z_{0}\right)\left[a+z_{0} p^{\prime}\left(z_{0}\right)\right]}{a+p^{2}\left(z_{0}\right)+z_{0} p^{\prime}\left(z_{0}\right)}\right]^{2}=\operatorname{Re}\left[\frac{i \lambda(a-k \lambda)}{a-\lambda^{2}-k \lambda}\right]^{2}=-\left[\frac{\lambda(a-k \lambda)}{a-\lambda^{2}-k \lambda}\right]^{2} \leq 0,
$$

which is a contradiction with the assumption (2.5).

(ii) For the case $p\left(z_{0}\right)=0$, using the fact that $a<\frac{1}{2}$ we have

$$
a+p^{2}\left(z_{0}\right)+z_{0} p^{\prime}\left(z_{0}\right)=a-\frac{l}{2} \neq 0,
$$

hence

$$
\operatorname{Re}\left[\frac{p\left(z_{0}\right)\left[a+z_{0} p^{\prime}\left(z_{0}\right)\right]}{a+p^{2}\left(z_{0}\right)+z_{0} p^{\prime}\left(z_{0}\right)}\right]^{2}=0,
$$

which contradicts the assumption 2.5).

From the two which discussed above it follows that $\operatorname{Re} p(z)>0$ for all $z \in \mathbb{U}$.

Taking $f \in \mathcal{A}$ and $p:=f^{\prime}$, and $p(z):=\frac{z f^{\prime}(z)}{f(z)}$ in Theorem 2.3 we obtain the next two special cases that represent sufficient condition for the close-to-convexity and starlikeness, respectively:

Corollary 2.5. If $f \in \mathcal{A}$, with $f^{\prime \prime}(0) \neq 0$, and satisfies

$$
\operatorname{Re}\left[\frac{f^{\prime}(z)\left[a+z f^{\prime \prime}(z)\right]}{a+\left[f^{\prime 2}+z f^{\prime \prime}(z)\right.}\right]^{2}>0, z \in \mathbb{U}, \quad \text { for some } a \in\left(-\infty, \frac{1}{2}\right) \backslash\{-1,0\},
$$


then

$$
\operatorname{Re} f^{\prime}(z)>0, z \in \mathbb{U} .
$$

Corollary 2.6. If $f \in \mathcal{A}$, with $f^{\prime \prime}(0) \neq 0$, and satisfies

$$
\operatorname{Re}\left[\frac{a+\frac{z f^{\prime}(z)}{f(z)}\left(1+\frac{z f^{\prime \prime}(z)}{f^{\prime}(z)}-\frac{z f^{\prime}(z)}{f(z)}\right)}{1+a \frac{f(z)}{z f^{\prime}(z)}+\frac{z f^{\prime \prime}(z)}{f^{\prime}(z)}}\right]^{2}>0, z \in \mathbb{U},
$$

for some $a \in\left(-\infty, \frac{1}{2}\right) \backslash\{-1,0\}$, then

$$
\operatorname{Re} \frac{z f^{\prime}(z)}{f(z)}>0, z \in \mathbb{U} .
$$

Theorem 2.4. Let $p$ be an analytic function in $\mathbb{U}$, with $p(0)=1, p^{\prime}(0) \neq 0$, that satisfies

$$
\operatorname{Re}\left[\frac{p(z)\left[a+z p^{\prime}(z)\right]}{a+\frac{z p^{\prime}(z)}{p^{2}(z)}}\right]^{2}>0, z \in \mathbb{U}, \quad \text { for some } a \in \mathbb{R}
$$

Then

$$
\operatorname{Re} p(z)>0, z \in \mathbb{U} .
$$

Proof. Suppose that there exists a point $z_{0} \in \mathbb{U}$ such that

$$
\operatorname{Re} p(z)>0, \text { for }|z|<\left|z_{0}\right|
$$

and

$$
\operatorname{Re} p\left(z_{0}\right)=0 \text {. }
$$

By using Lemma 1.1 for $m=1$, it follows that

$$
p\left(z_{0}\right)=i \lambda, \lambda \in \mathbb{R},
$$

and

$$
z_{0} p^{\prime}\left(z_{0}\right)=\left\{\begin{array}{lll}
i k p\left(z_{0}\right), & \text { when } & p\left(z_{0}\right) \neq 0 \\
-l / 2, & \text { when } & p\left(z_{0}\right)=0
\end{array}= \begin{cases}-k \lambda, & \text { when } \lambda \neq 0 \\
-l / 2, & \text { when } \lambda=0,\end{cases}\right.
$$

for some $k \geq 1, l \geq 1$.

(i) For the case $p\left(z_{0}\right) \neq 0$, that is $\lambda \neq 0$, suppose that

$$
a+\frac{z_{0} p^{\prime}\left(z_{0}\right)}{p^{2}\left(z_{0}\right)}=a+\frac{k}{\lambda}=0 .
$$

( $\alpha$ ) If

$$
p\left(z_{0}\right)\left[a+z_{0} p^{\prime}\left(z_{0}\right)\right]=i \lambda(a-k \lambda) \neq 0,
$$


then $z_{0} \in \mathbb{U}$ will be a double pole for the function

$$
\left[\frac{p(z)\left[a+z p^{\prime}(z)\right]}{a+\frac{z p^{\prime}(z)}{p^{2}(z)}}\right]^{2}
$$

and therefore, in any neighborhood $U\left(z_{0} ; \rho\right):=\{z \in \mathbb{C}:|z|<\rho\} \subset \mathbb{U}$ of the pole $z_{0}$ there exists at least a $z_{\rho} \in U\left(z_{0} ; \rho\right)$ such that have

$$
\left[\frac{p\left(z_{\rho}\right)\left[a+z_{\rho} p^{\prime}\left(z_{\rho}\right)\right]}{a+\frac{z_{\rho} p^{\prime}\left(z_{\rho}\right)}{p^{2}\left(z_{\rho}\right)}}\right]^{2}<0
$$

which contradicts the assumption 2.7 .

( $\beta)$ If

$$
p\left(z_{0}\right)\left[a+z_{0} p^{\prime}\left(z_{0}\right)\right]=i \lambda(a-k \lambda)=0,
$$

then $a=k \lambda$ and from 2.8 it follows that $k=0$ or $\lambda^{2}=-1$, which contradicts the facts $k \geq 1$ and $\lambda \in \mathbb{R}$.

Therefore, from $(\alpha)$ and $(\beta)$ we deduce that the assumption 2.7 implies that the function

is analytic in $\mathbb{U}$, and

$$
\left[\frac{p(z)\left[a+z p^{\prime}(z)\right]}{\left.a+\frac{z p^{\prime}(z)}{p^{2}(z)}\right]^{2}}\right.
$$

$$
\operatorname{Re}\left[\frac{p\left(z_{0}\right)\left[a+z_{0} p^{\prime}\left(z_{0}\right)\right]}{a+\frac{z_{0} p^{\prime}\left(z_{0}\right)}{p^{2}\left(z_{0}\right)}}\right]^{2}=\operatorname{Re}\left[\frac{i \lambda(a-k \lambda)}{a+\frac{k}{\lambda}}\right]^{2}=-\left[\frac{\lambda(a-k \lambda)}{a+\frac{k}{\lambda}}\right]^{2} \leq 0
$$

which is a contradiction with the assumption (2.7).

(ii) For the case $p\left(z_{0}\right)=0$, since

$$
\left[\frac{p(z)\left[a+z p^{\prime}(z)\right]}{a+\frac{z p^{\prime}(z)}{p^{2}(z)}}\right]^{2}=\left[\frac{p^{3}(z)\left[a+z p^{\prime}(z)\right]}{a p^{2}(z)+z p^{\prime}(z)}\right]^{2}
$$

it follows that

$$
\operatorname{Re}\left[\frac{p^{3}\left(z_{0}\right)\left[a+z_{0} p^{\prime}\left(z_{0}\right)\right]}{a p^{2}\left(z_{0}\right)+z_{0} p^{\prime}\left(z_{0}\right)}\right]^{2}=0
$$

which contradicts the assumption (2.7). 
Concluding, from the two cases we discussed above it follows that $\operatorname{Re} p(z)>0$ for all $z \in \mathbb{U}$.

Replacing $p:=f^{\prime}$, and $p(z):=\frac{z f^{\prime}(z)}{f(z)}$ where $f \in \mathcal{A}$ in Theorem 2.4 we obtain the next two special cases that represent sufficient condition for the close-to-convexity and starlikeness, respectively:

Corollary 2.7. If $f \in \mathcal{A}$, with $f^{\prime \prime}(0) \neq 0$, and satisfies

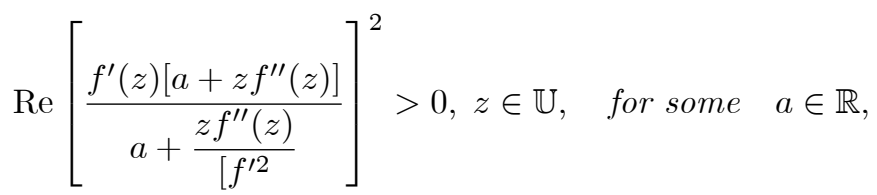

then,

$$
\operatorname{Re} f^{\prime}(z)>0, z \in \mathbb{U} \text {. }
$$

Corollary 2.8. If $f \in \mathcal{A}$, with $f^{\prime \prime}(0) \neq 0$, and satisfies

$\operatorname{Re}\left[\frac{\frac{z f^{\prime}(z)}{f(z)}\left[a+\frac{z f^{\prime}(z)}{f(z)}\left(1+\frac{z f^{\prime \prime}(z)}{f^{\prime}(z)}-\frac{z f^{\prime}(z)}{f(z)}\right)\right]}{a+\frac{f(z)}{z f^{\prime}(z)}\left(1+\frac{z f^{\prime \prime}(z)}{f^{\prime}(z)}-\frac{z f^{\prime}(z)}{f(z)}\right)}\right]^{2}>0, z \in \mathbb{U}$, for some $a \in \mathbb{R}$,

then,

$$
\operatorname{Re} \frac{z f^{\prime}(z)}{f(z)}>0, z \in \mathbb{U} .
$$

Remark 2.1. (1) For $g \in \mathcal{S}^{*}$ and $f \in \mathcal{A}$, such that $2 f^{\prime \prime}(0) \neq g^{\prime \prime}(0)$, setting $p(z):=\frac{z f^{\prime}(z)}{g(z)}$ in the above theorems we will obtain sufficient condition for close-to-convexity.

(2) For $f \in \mathcal{A}$, with $f^{\prime \prime}(0) \neq 0$, setting $p(z):=\frac{f(z)}{z}$ in the above theorems we will obtain sufficient condition for the functions $f$ to satisfy the inequality $\operatorname{Re} \frac{f(z)}{z}>0, z \in \mathbb{U}$.

\section{Coefficient Bounds}

We begin by deriving upper bounds for the general Taylor-Maclaurin coefficients $\left|a_{n}\right|$ for $n \geq 3$ of the functions belonging in the class $\mathcal{B}_{\Sigma}^{q, \mu}(\beta, \lambda, h)$, and next we will find estimates for the initial coefficient $\left|a_{2}\right|$.

Using the Faber polynomial expansion of functions $f \in \mathcal{S}$ of the form 1.1), the coefficients of its inverse map $g=f^{-1}$ may be expressed as follows (see for details 
[1] and [2])

$$
g(w)=f^{-1}(w)=w+\sum_{n=2}^{\infty} \frac{1}{n} K_{n-1}^{-n}\left(a_{2}, a_{3}, \ldots, a_{n}\right) w^{n}
$$

where

$$
\begin{aligned}
K_{n-1}^{-n}= & \frac{(-n) !}{(-2 n+1) !(n-1) !} a_{2}^{n-1}+\frac{(-n) !}{(2(-n+1)) !(n-3) !} a_{2}^{n-3} a_{3} \\
& +\frac{(-n) !}{(-2 n+3) !(n-4) !} a_{2}^{n-4} a_{4}+\frac{(-n) !}{(2(-n+2)) !(n-5) !} a_{2}^{n-5}\left[a_{5}+(-n+2) a_{3}^{2}\right] \\
& +\frac{(-n) !}{(-2 n+5) !(n-6) !} a_{2}^{n-6}\left[a_{6}+(-2 n+5) a_{3} a_{4}\right]+\sum_{j \geq 7} a_{2}^{n-j} V_{j}
\end{aligned}
$$

such that $V_{j}(7 \leq j \leq n)$ is a homogeneous polynomial in the variables $a_{2}, a_{3}, \ldots, a_{n}$, and the expressions such as (for example) $(-n)$ ! are to be interpreted symbolically by

$$
\begin{aligned}
(-n) ! & \equiv \Gamma(1-n):=(-n)(-n-1)(-n-2) \ldots, \\
\text { with } n & \in \mathbb{N}_{0}:=\mathbb{N} \cup\{0\}, \mathbb{N}:=\{1,2,3, \ldots\} .
\end{aligned}
$$

In particular, the first three terms of $K_{n-1}^{-n}$ are given by

$$
K_{1}^{-2}=-2 a_{2}, \quad K_{2}^{-3}=3\left(2 a_{2}^{2}-a_{3}\right) \quad \text { and } \quad K_{3}^{-4}=-4\left(5 a_{2}^{3}-5 a_{2} a_{3}+a_{4}\right) .
$$

In general, for any $p$ real value the expansion of $K_{n}^{p}$ is given below (see for details, [1, 29]; see also [2, p. 349])

$$
K_{n}^{p}=p a_{n+1}+\frac{p(p-1)}{2} D_{n}^{2}+\frac{p !}{(p-3) ! 3 !} D_{n}^{3}+\cdots+\frac{p !}{(p-n) ! n !} D_{n}^{n},
$$

where $D_{n}^{p}=D_{n}^{p}\left(a_{2}, a_{3}, \ldots, a_{n+1}\right)$ (see for details [29]). We also have

$$
D_{n}^{m}\left(a_{2}, a_{3}, \ldots, a_{n+1}\right)=\sum_{n=1}^{\infty} \frac{m !\left(a_{2}\right)^{\mu_{1}} \cdot \ldots \cdot\left(a_{n+1}\right)^{\mu_{n}}}{\mu_{1} ! \cdot \ldots \cdot \mu_{n} !}
$$

where the sum is taken over all nonnegative integers $\mu_{1}, \ldots, \mu_{n}$ satisfying the conditions

$$
\left\{\begin{array}{l}
\mu_{1}+\mu_{2}+\cdots+\mu_{n}=m \\
\mu_{1}+2 \mu_{2}+\cdots+n \mu_{n}=n
\end{array}\right.
$$

It is clear that $D_{n}^{n}\left(a_{2}, a_{3}, \ldots, a_{n+1}\right)=a_{2}^{n}$.

Theorem 3.1. Let the function $f \in \mathcal{B}_{\Sigma}^{q, \mu}(\beta, \lambda, h)$ be given by 1.1) with the power expansion of the function $h$ given by

$$
h(z)=1+\sum_{n=1}^{\infty} B_{n} z^{n}, z \in \mathbb{U},
$$


and suppose that $B_{1} \neq 0$. If $a_{k}=0$ for $2 \leq k \leq n-1$, where $n \geq 3$, then

$$
\left|a_{n}\right| \leq \frac{\left|B_{1}\right| \cos \beta}{[\lambda+(n-1)] \Theta_{n}} .
$$

Proof. For $f \in \mathcal{B}_{\Sigma}^{q, \mu}(\beta, \lambda, h)$ given by (1.1), using the relations (1.6) and (1.7) from [2, page 344] we have

$e^{i \beta}\left(\frac{z^{1-\lambda}\left(\Omega_{q}^{\mu} f(z)\right)^{\prime}}{\left[\Omega_{q}^{\mu} f(z)\right]^{1-\lambda}}\right)=e^{i \beta}\left(1+\sum_{n=2}^{\infty}\left(1+\frac{n-1}{\lambda}\right) K_{n-1}^{\lambda}\left(\Theta_{2} a_{2}, \Theta_{3} a_{3}, \ldots, \Theta_{n} a_{n}\right) z^{n-1}\right)$,

and for its inverse map $g=f^{-1}$, according to the expansion formula 1.2 we have

$e^{i \beta}\left(\frac{w^{1-\lambda}\left(\Omega_{q}^{\mu} g(w)\right)^{\prime}}{\left[\Omega_{q}^{\mu} g(w)\right]^{1-\lambda}}\right)=e^{i \beta}\left(1+\sum_{n=2}^{\infty}\left(1+\frac{n-1}{\lambda}\right) K_{n-1}^{\lambda}\left(\Theta_{2} b_{2}, \Theta_{3} b_{3}, \ldots, \Theta_{n} b_{n}\right) w^{n-1}\right)$,

where $b_{n}=\frac{1}{n} K_{n-1}^{-n}\left(a_{2}, a_{3}, \ldots, a_{n}\right), n=2,3, \ldots$ are defined by 3.1).

Furthermore, since $f \in \mathcal{B}_{\Sigma}^{q, \mu}(\beta, \lambda, h)$, from the definition of the subordination there exist two Schwartz functions $u, v: \mathbb{U} \rightarrow \mathbb{U}$ of the form $u(z)=\sum_{n=1}^{\infty} p_{n} z^{n}$, $v(z)=\sum_{n=1}^{\infty} q_{n} z^{n}$, such that

$$
e^{i \beta}\left(\frac{z^{1-\lambda}\left(\Omega_{q}^{\mu} f(z)\right)^{\prime}}{\left[\Omega_{q}^{\mu} f(z)\right]^{1-\lambda}}\right)=h(u(z)) \cos \beta+i \sin \beta
$$

and

$$
e^{i \beta}\left(\frac{w^{1-\lambda}\left(\Omega_{q}^{\mu} g(w)\right)^{\prime}}{\left[\Omega_{q}^{\mu} g(w)\right]^{1-\lambda}}\right)=h(v(w)) \cos \beta+i \sin \beta .
$$

Moreover, from (3.3) we have

$$
h(u(z))=1+B_{1} p_{1} z+\left(B_{1} p_{2}+B_{2} p_{1}^{2}\right) z^{2}+\cdots=1+\sum_{n=1}^{\infty} \sum_{k=1}^{n} B_{k} D_{n}^{k}\left(p_{1}, p_{2}, \ldots, p_{n}\right) z^{n},
$$

and

$$
h(v(w))=1+\sum_{n=1}^{\infty} \sum_{k=1}^{n} B_{k} D_{n}^{k}\left(q_{1}, q_{2}, \ldots, q_{n}\right) w^{n} .
$$

Equating the corresponding coefficients of (3.6) and (3.7) we get, respectively,

$$
e^{i \beta}\left(1+\frac{n-1}{\lambda}\right) K_{n-1}^{\lambda}\left(\Theta_{2} a_{2}, \Theta_{3} a_{3}, \ldots, \Theta_{n} a_{n}\right)=\sum_{k=1}^{n-1} B_{k} D_{n-1}^{k}\left(p_{1}, p_{2}, \ldots, p_{n-1}\right) \cos \beta
$$


and

$$
e^{i \beta}\left(1+\frac{n-1}{\lambda}\right) K_{n-1}^{\lambda}\left(\Theta_{2} b_{2}, \Theta_{3} b_{3}, \ldots, \Theta_{n} b_{n}\right)=\sum_{k=1}^{n-1} B_{k} D_{n-1}^{k}\left(q_{1}, q_{2}, \ldots, q_{n-1}\right) \cos \beta .
$$

We observe that if $a_{k}=0$ for all $2 \leq k \leq n-1$, by the definition of $K_{n}^{p}$ we have $b_{n}=-a_{n}$, and since $B_{1} \neq 0$ we have $p_{1}=\cdots=p_{n-2}=0$ and $q_{1}=\cdots=q_{n-2}=0$. Hence from (3.8) and 3.9) we obtain, respectively,

$$
[\lambda+(n-1)] e^{i \beta} \Theta_{n} a_{n}=B_{1} p_{n-1} \cos \beta
$$

and

$$
-[\lambda+(n-1)] e^{i \beta} \Theta_{n} a_{n}=B_{1} q_{n-1} \cos \beta .
$$

Taking the modules of either of the above two equalities and using Lemma 1.2 we obtain our result.

Theorem 3.2. Let the function $f \in \mathcal{B}_{\Sigma}^{q, \mu}(\beta, \lambda, h)$ be given by (1.1). Then

$$
\left|a_{2}\right| \leq \frac{\left|B_{1}\right| \sqrt{2\left|B_{1}\right|} \cos \beta}{\sqrt{\left|B_{1}\right|^{2}\left|(\lambda-1)(\lambda+2) \Theta_{2}^{2}+2(\lambda+2) \Theta_{3}\right| \cos \beta+2\left(\left|B_{1}\right|-\left|B_{2}\right|\right)(1+\lambda)^{2} \Theta_{2}^{2}}},
$$

for those values of all the parameters such that the denominator is not zero.

Proof. If we set $n=2$ and $n=3$ in (3.8) and (3.9), respectively, we obtain

$$
\begin{aligned}
& e^{i \beta}(1+\lambda) \Theta_{2} a_{2}=B_{1} p_{1} \cos \beta \\
& e^{i \beta}\left[\frac{(\lambda-1)(\lambda+2)}{2} \Theta_{2}^{2} a_{2}^{2}+(\lambda+2) \Theta_{3} a_{3}\right]=\left(B_{1} p_{2}+B_{2} p_{1}^{2}\right) \cos \beta \\
& -e^{i \beta}(1+\lambda) \Theta_{2} a_{2}=B_{1} q_{1} \cos \beta \\
& e^{i \beta}\left[\left(\frac{(\lambda-1)(\lambda+2)}{2} \Theta_{2}^{2}+2(\lambda+2) \Theta_{3}\right) a_{2}^{2}-(\lambda+2) \Theta_{3} a_{3}\right] \\
= & \left(B_{1} q_{2}+B_{2} q_{1}^{2}\right) \cos \beta .
\end{aligned}
$$

From 3.10 and 3.12 we get

$$
p_{1}=-q_{1},
$$

then, adding (3.11) and (3.13) and according to (3.14) we obtain

$$
e^{i \beta}\left[(\lambda-1)(\lambda+2) \Theta_{2}^{2}+2(\lambda+2) \Theta_{3}\right] a_{2}^{2}=B_{1}\left(p_{2}+\frac{B_{2}}{B_{1}} p_{1}^{2}+q_{2}+\frac{B_{2}}{B_{1}} q_{1}^{2}\right) \cos \beta .
$$

From 3.10 , using Lemma 1.3 we have

$$
\begin{aligned}
& \left|(\lambda-1)(\lambda+2) \Theta_{2}^{2}+2(\lambda+2) \Theta_{3}\right|\left|a_{2}\right|^{2} \leq\left|B_{1}\right|\left(\left|p_{2}+\frac{B_{2}}{B_{1}} p_{1}^{2}\right|+\left|q_{2}+\frac{B_{2}}{B_{1}} q_{1}^{2}\right|\right) \cos \beta \\
\leq & 2\left|B_{1}\right|\left(1+\frac{\left|B_{2}\right|-\left|B_{1}\right|}{\left|B_{1}\right|}\left|p_{1}^{2}\right|\right) \cos \beta=2\left|B_{1}\right|\left[1+\frac{\left(\left|B_{2}\right|-\left|B_{1}\right|\right)(1+\lambda)^{2} \Theta_{2}^{2}\left|a_{2}^{2}\right|}{\left|B_{1}\right|^{3} \cos ^{2} \beta}\right] \cos \beta .
\end{aligned}
$$


After some simple computations, from the above inequality we have

$$
\begin{gathered}
{\left[\left|B_{1}\right|^{2}\left|(\lambda-1)(\lambda+2) \Theta_{2}^{2}+2(\lambda+2) \Theta_{3}\right| \cos \beta+2\left(\left|B_{1}\right|-\left|B_{2}\right|\right)(1+\lambda)^{2} \Theta_{2}^{2}\right]\left|a_{2}\right|^{2}} \\
\leq 2\left|B_{1}\right|^{3} \cos ^{2} \beta
\end{gathered}
$$

which implies our result.

Remark 3.1. (1) The bound for $\left|a_{2}\right|$ from Theorem 3.2 is smaller than the estimate obtained by Murugusundaramoorthy et al. in [22, Theorem 2.1].

(2) Letting $h(z)=\frac{1+A z}{1+B z},-1 \leq B<A \leq 1$, in Theorem 3.2 we obtain an improvement of the estimate for $\left|a_{2}\right|$ obtained by Murugusundaramoorthy et al. in [22, Theorem 3.1], and it is presented in the next example.

(3) Setting $h(z)=\frac{1+(1-2 \alpha) z}{1-z}, 0 \leq \alpha<1$, in Theorem 3.2, we obtain an improvement of the estimate for $\left|a_{2}\right|$ obtained by Murugusundaramoorthy et al. in [22, Theorem 4.1], like we will show in Example 3.2.

(4) By setting $\lambda=1, \beta=\mu=0$, and $q \rightarrow 1^{-}$in Theorem 3.2 we get $\Theta_{n}=$ 1 , hence we obtain an improvement of the estimate for $\left|a_{2}\right|$ obtained by Algahtani in [4, Theorem 2.3].

(5) Taking $\lambda=\beta=\mu=0$ and $q \rightarrow 1^{-}$in Theorem 3.2 we get $\Theta_{n}=1$, hence we obtain an improvement of the estimate for $\left|a_{2}\right|$ obtained by Algahtani in [4, Theorem 2.6].

Example 3.1. Let the function $f \in \mathcal{B}_{\Sigma}^{q, \mu}\left(\beta, \lambda, \frac{1+A z}{1+B z}\right)$ be given by 1.1), where $-1 \leq B<A \leq 1$. If $a_{k}=0$ for $2 \leq k \leq n-1$, where $n \geq 3$, then

$$
\left|a_{n}\right| \leq \frac{(A-B) \cos \beta}{[\lambda+(n-1)] \Theta_{n}} .
$$

Example 3.2. Let the function $f \in \mathcal{B}_{\Sigma}^{q, \mu}\left(\beta, \lambda, \frac{1+(1-2 \alpha) z}{1-z}\right)$ be given by 1.1), where $0 \leq \alpha<1$. If $a_{k}=0$ for $2 \leq k \leq n-1$, where $n \geq 3$, then

$$
\left|a_{n}\right| \leq \frac{2(1-\alpha) \cos \beta}{[\lambda+(n-1)] \Theta_{n}} .
$$

\section{Conclusion}

In the final section, using the Faber polynomial expansion we found upper bounds for $\left|a_{n}\right|(n \geq 3)$ coefficients of functions in the class defined by Definition 1.1, and then we obtained an estimate for the initial coefficients $\left|a_{2}\right|$ for the functions of this class. Thus, regarding the proofs of the Theorems 3.1 and 3.2, this technique can be applied for all classes that are defined similarly to the Definition 1.1 in diverse papers enhancing their outcomes (see for example [3, 10, 21, 23, 28] and references therein). 
Acknowledgments. The authors thank from the Najafabad Branch, Islamic Azad University for their financial support.

\section{REFERENCES}

[1] Airault, H. and Bouali, A. Differential calculus on the Faber polynomials, Bull. Sci. Math., 130 (2006), 179-222.

[2] Airault, H. and Ren, J. An algebra of differential operators and generating functions on the set of univalent functions, Bull. Sci. Math., 126 (2002), 343-367.

[3] Alamoush, A. G. and Darus, M. On coefficient estimates for bi-univalent functions of FoxWright functions, Far East J. Math. Sci. (FJMS), 89 (2014), 249-262.

[4] Algahtani, O. Estimates of initial coefficients for certain subclasses of bi-univalent functions involving quasi-subordination, J. Nonlinear Sci. Appl., 10 (2017), 1004-1011.

[5] Aouf, M. K. and Bulboacă, T. Subordination and superordination properties of multivalent functions defined by certain integral operator, J. Franklin Institute, 347 (3) (2010), 641-653.

[6] Aouf, M. K. and Dziok, J., Distortion and convolution theorems for operators of generalized fractional calculus involving Wright function, J. Appl. Anal., 14 (2008), 183-192.

[7] Bukhari, S. Z. H., Bulboacă, T., Shabbir, M. S., Subordination and superordination results for analytic functions with respect to symmetrical points, Quaest. Math., 41 (1) (2018) 65-79.

[8] Bulboacă, T. On some classes of differential subordinations, Studia Univ. Babeş-Bolyai Math., 31 (1986), 45-50.

[9] Bulboacă, T. On some classes of differential subordinations (II), Studia Univ. Babę̧-Bolyai Math., 31 (1986), 13-21.

[10] Bulut, S. Coefficient estimates for initial Taylor-Maclaurin coefficients for a subclass of analytic and bi-univalent functions defined by Al-Oboudi differential operator, Sci. World J., (2013), Art. ID 171039, 6 pages.

[11] Duren, P. L. Univalent Functions, Grundlehren der Mathematischen Wissenschaften, 259, Springer, New York, (1983).

[12] El-Ashwah, R. M., Aouf, M. K., Bulboacă, T. Differential subordinations for classes of meromorphic $p$-valent functions defined by multiplier transformations, Bull. Aust. Math. Soc., 83 (2011), 353-368.

[13] Kanas, S., Tudor, A. E. Differential subordinations and harmonic means, Bull. Malays. Math. Sci. Soc. (2), 38 (2015), 1243-1253.

[14] Keogh, F. R., Merkes, E. P. A coeffcient inequality for certain classes of analytic functions, Proc. Amer. Math. Soc., 20 (1969), 8-12.

[15] Kumar, S., Ravichandran, V. Subordinations for functions with positive real part, Complex Anal. Oper. Theory, (2017), 1-15. DOI:10.1007/s11785-017-0690-4

[16] Hamidi, S. G., Jahangiri, J. M. Faber polynomial coefficient estimates for analytic bi-closetoconvex functions, C. R. Math. Acad. Sci. Paris, 352 (2014), 17-20.

[17] Hamidi, S. G., Jahangiri, J. M. Faber polynomial coefficients of bi-subordinate functions, $C$. R. Math. Acad. Sci. Paris, 354 (2016), 365-370.

[18] Miller, S. S., Mocanu, P. T. Second-order differential inequalities in the complex plane, J. Math. Anal. Appl., 65 (1978), 298-305.

[19] Nunokawa, M., Sokół, J. On multivalent starlike functions and Ozaki condition, Complex Var. Elliptic Equ., (2018), 1-15, DOI:10.1080/17476933.2017.1419209

[20] Nunokawa, M., Sokół, J. On some differential subordinations, Studia Sci. Math. Hungar., 54 (2017), 436-445.

[21] Murugusundaramoorthy, G. Coefficient estimates of bi-Bazilević functions defined by Srivastava-Attiya operator, Matematiche (Catania), 69 (2014), 43-56.

[22] Murugusundaramoorthy, G., Janani, T., Purohit, S. D. Coefficient estimate of bi-Bazilević functions associated with fractional $q$-calculus operators, Fund. Inform., 151 (2017), 49-62. 
[23] Orhan, H., Magesh, N., Balaji, V. K. Initial coeffcient Bounds for a general class of biunivalent functions, Filomat, 29 (2015), 1259-1267.

[24] Owa, S., Srivastava, H. M. Univalent and starlike generalized hypergeometric functions, Canad. J. Math., 39 (1987), 1057-1077.

[25] Purohit, S. D., Raina, R. K. Certain subclasses of analytic functions associated with fractional q-calculus operators, Math. Scand., 10 (2011), 55-70.

[26] Srivatava, H. M., Aouf, M. K. Some applications of fractional calculus operators to certain subclasses of prestarlike functions with negative coefficients, Comput. Math. Appl., 30 (1) (1995), 53-61.

[27] Srivastava, H. M., Gaboury, S., Ghanim, F. Coefficient estimates for a general subclass of analytic and bi-univalent functions of the Ma-Minda type, RACSAM Rev. R. Acad. Cienc. Exactas Fís. Nat. Ser. A Mat., (2017), DOI:10.1007/s13398-017-0416-5

[28] Srivastava, H. M., Murugusundaramoorthy, G., Vijaya, K. Coefficient estimates for some families of bi-Bazilevic functions of the Ma-Minda type involvig the Hohlov operator, $J$. Classical Anal., 2 (2013), 167-181.

[29] Todorov, P. G., On the Faber polynomials of the univalent functions of class $\Sigma$, J. Math. Anal. Appl., 162 (1991), 268-276.

[30] Zireh, A., Adegani, E. A., Bulut, S. Faber polynomial coefficient estimates for a comprehensive subclass of analytic bi-univalent functions defined by subordination, Bull. Belg. Math. Soc. Simon Stevin, 23 (2016), 487-504.

Current address, M. Jafari: (Corresponding author) Department of Mathematics, Najafabad

Branch, Islamic Azad University, Najafabad, Iran

E-mail address: mostafajafari83@gmail.com

ORCID Address: http://orcid.org/0000-0002-2144-1097

Current address, T. Bulboacă: Faculty of Mathematics and Computer Science, Babeş-Bolyai University, 400084 Cluj-Napoca, Romania

E-mail address: bulboaca@math.ubbcluj.ro

ORCID Address: http://orcid.org/0000-0001-8026-218X

Current address, A. Zireh: Faculty of Mathematical Sciences, University of Shahrood, P.O.Box 316-36155, Shahrood, Iran

E-mail address: azireh@gmail.com

ORCID Address: http://orcid.org/0000-0002-3405-853X

Current address, E. Analouei Adegani: Faculty of Mathematical Sciences, University of Shahrood, P.O.Box 316-36155, Shahrood, Iran

E-mail address: analoey.ebrahim@gmail.com

ORCID Address: http://orcid.org/0000-0001-8026-218X 